\title{
Effect of Age and Severity of Facial Palsy on Taste Thresholds in Bell's Palsy Patients
}

\author{
Jung Min Park', Myung Gu Kim², Junyang Jung ${ }^{3}$, Sung Su Kim \\ A Ra Jung ${ }^{1}$, Sang Hoon Kim ${ }^{1}$, and Seung Geun Yeo ${ }^{1}$ \\ ${ }^{1}$ Department of Otorhinolaryngology-Head and Neck Surgery, School of Medicine, Kyung Hee University, Seoul, \\ 2Department of Otorhinolaryngology-Head and Neck Surgery, Samsung Changwon Hospital, \\ Sungkyunkwan University School of Medicine, Changwon, \\ ${ }^{3}$ Department of Anatomy, School of Medicine, Kyung Hee University, Seoul, \\ ${ }^{4}$ Department of Biochemistry and Molecular Biology, Medical Science and Engineering Research Center for Bioreaction \\ to Reactive Oxygen Species, BK-21, School of Medicine, Kyung Hee University, Seoul, Korea
}

\section{Received August 17, 2016 \\ Revised November 15, 2016 \\ Accepted December 92016}

Background and Objectives: To investigate whether taste thresholds, as determined by electrogustometry (EGM) and chemical taste tests, differ by age and the severity of facial palsy in patients with Bell's palsy. Subjects and Methods: This study included 29 patients diagnosed with Bell's palsy between January 2014 and May 2015 in our hospital. Patients were assorted into age groups and by severity of facial palsy, as determined by HouseBrackmann Scale, and their taste thresholds were assessed by EGM and chemical taste tests. Results: EGM showed that taste thresholds at four locations on the tongue and one location on the central soft palate, $1 \mathrm{~cm}$ from the palatine uvula, were significantly higher in Bell's palsy patients than in controls $(p<0.05)$. In contrast, chemical taste tests showed no significant differences in taste thresholds between the two groups ( $p>0.05$ ). The severity of facial palsy did not affect taste thresholds, as determined by both EGM and chemical taste tests ( $p>0.05$ ). The overall mean electrical taste thresholds on EGM were higher in younger Bell's palsy patients than in healthy subjects, with the difference at the back-right area of the tongue differing significantly $(p<0.05)$. In older individuals, however, no significant differences in taste thresholds were observed between Bell's palsy patients and healthy subjects ( $p>0.05)$. Conclusions: Electrical taste thresholds were higher in Bell's palsy patients than in controls. These differences were observed in younger, but not in older, individuals.

J Audiol Otol 2017;21(1):16-21

\section{Introduction}

The most common symptoms in patients with Bell's palsy include facial palsy and facial asymmetry. This condition, however, is sometimes accompanied by other symptoms, including otalgia, taste loss, dry eyes/eye discomfort, tinnitus, hearing loss, and dizziness. Bell's palsy patients who recover incompletely from their main symptoms after treatment are often dissatisfied with their outcomes, but little is known

This is an Open Access article distributed under the terms of the Creative Commons Attribution Non-Commercial License (http://creativecommons.org/licenses/by-nc/4.0/) which permits unrestricted non-commercial use, distribution, and reproduction in any medium, provided the original work is properly cited. about the link between treatment and accompanying residual symptoms and patient satisfaction [1].

The facial nerves have multiple functions, including: 1) the control of muscles responsible for facial expressions, via motor fibers, 2) the control of secretions by the lacrimal and salivary glands, via parasympathetic fibers, 3) the control of taste buds in the anterior two-thirds of the tongue, via special sensory nerve fibers, and 4) the control of general sensory nerve fibers that affect the auricles, the posterior wall of the external auditory canals, the ear lobes, and deep sensations of facial soft tissue. The special sensory nerve fibers of the facial nerve convey taste sensations from the anterior twothirds of the tongue via the chorda tympani nerve and genic- 
ulate ganglion to the nucleus tractus solitaries in the medulla oblongata. Thus, paralysis of the facial nerve may lead to dysgeusia in the anterior two-thirds of the tongue [2].

Factors affecting sensitivity to tastes include smell disorders, smoking, ingestion of alcohol, medications, degeneration with aging, endocrine changes in climacteric and pregnant women, mental and psychological factors, and xerostomia. Other factors include chronic diseases of the upper respiratory tract and laryngopharynx, chewing disorders, salivary gland disorders, poor dental hygiene and malocclusion of dental prostheses [3-5]. Many factors affecting taste acuity have been analyzed, including aging, foods, upper respiratory tract infections, olfactory sense, drinking alcohol, medications, trauma, head injury, chemical damage, accident, heavy metal poisoning, endocrine-metabolic disorders, neurodegenerative diseases, neurotransmitters, tumors, congenital abnormalities, and lifestyle patterns. To date, however, few studies have utilized electrogustometry (EGM) and chemical taste tests to assess the reduced sense of taste in Bell's palsy patients [6,7]. This study therefore compared taste thresholds in Bell's palsy patients and healthy subjects, as well as the effects on taste of age and the severity of facial paralysis.

\section{Subjects and Methods}

The study included 29 subjects diagnosed with Bell's palsy in our hospital between January 2014 and May 2015; their degree of Bell's palsy was determined using the HouseBrackmann Scale. The control group consisted of 47 healthy volunteers without previous taste disorder or ENT diseases (IRB No. KMC 2014-05-108). Subjects in both groups were classified by age into those $<40$ and $\geq 40$ years. Subjects were evaluated by EGM (EG-IIB; Nagashima Medical Instrument Co., Tokyo, Japan) and chemical taste tests at within 14 days from the onset. Those who met any of the following criteria were excluded from the study: lack of a sense of hygiene; medical history of secretory, oral, degenerative neuronal, endocrine, cerebrovascular or psychological disease; innate abnormalities; or tumors. Patients suspected of neurological abnormalities and those with dysosmia or dysgeusia were also excluded. Participants were instructed not to eat or drink anything besides water at least one hour before testing.

EGM was used to measure electric threshold scores at four locations on the tongue, consisting of the left and right middle portions and the anterior and posterior borders of the circumvallate papillae; and at one location on the central soft palate, $1 \mathrm{~cm}$ from the palatine uvula (Fig. 1). EGM delivery of electric stimuli to these locations using a steel probe has been shown to produce a metallic, sour or bitter taste, with the lowest electric current defined as the threshold value. Before the start of testing, a stimulus greater than the threshold value was administered to ensure that every subject could recognize EGM stimuli. Stimulation was initiated at the lowest current $(3 \mu \mathrm{A})$ and was increased in 22 steps to a maximum of $400 \mu \mathrm{A}$ until the subjects recognized the stimulus.

Chemical tastes were performed at two locations, the midline of the tongue and the central soft palate, $1 \mathrm{~cm}$ above from the uvula. Five reagents were tested; sucrose for sweet taste, sodium chloride for salty taste, quinine-hydrochloride for bitter taste, tartaric acid for sour taste, and monosodium glutamate for savory taste (Fig. 2). Each reagent was tested at 10 concentrations. Several drops of each concentration of reagent were carefully placed on the two locations using a pipette. Subjects were instructed to indicate the taste threshold and determine the type of taste. To wash out the previous taste, the order of the chemical stimuli was randomized, the mouth was rinsed with tap water, and 1-2 minutes were allowed to elapse until the next test.

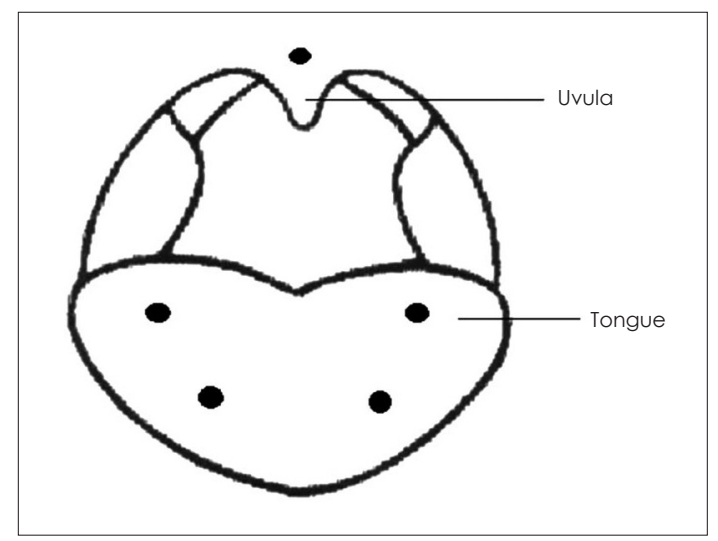

Fig. 1. Areas assessed by electrogustometry. Five areas (black circles) were evaluated, $2 \mathrm{~cm}$ lateral to the midline tongue tip, the lateral borders of the circumvallate papilla, and $1 \mathrm{~cm}$ above the uvula.

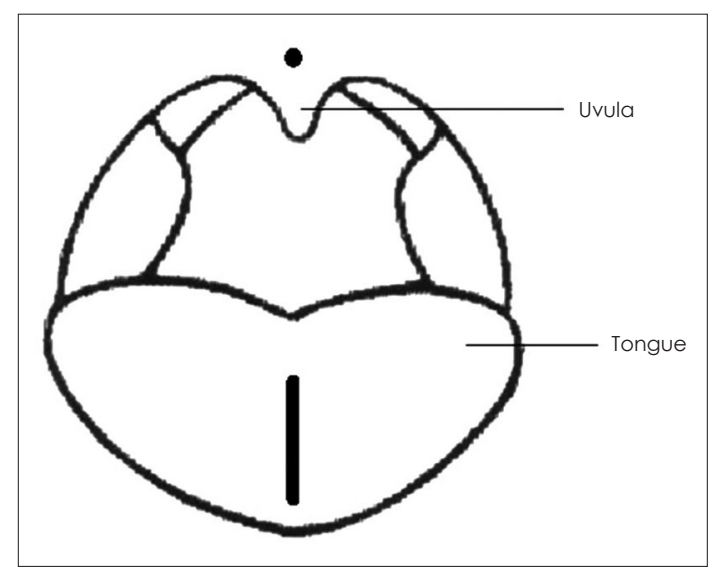

Fig. 2. Areas evaluated by chemical taste tests. These included the midline of the tongue and $1 \mathrm{~cm}$ above the uvula (black circles). 
All statistical analyses were performed using the Statistical Package for the Social Sciences, version 18.0 (SPSS Inc., Chicago, IL, USA). The electrical threshold values of the two groups were compared using Mann-Whitney U tests. The level of statistical significance was set at $p<0.05$.

\section{Results}

\section{Subjects}

The 29 patients in the Bell's palsy group comprised 8 males and 21 females, of mean age 41.3 years (range: $14-70$ years); these included 11 patients $<40$ years (mean age, 23.9 years) and 18 patients $\geq 40$ years (mean age, 52 years). In comparison, the 47 healthy subjects comprised 21 males and 26 females, of mean age 37.3 years (range: 18-76 years); these included 28 subjects $<40$ years (mean age, 28.4 years) and 19 subjects $\geq 40$ years (mean age, 50.4 years). There was no significant difference in age between control and facial palsy groups. ( $p>0.05)$. Of the 29 Bell's palsy patients, 17 had right-sided and 12 had left-sided Bell's palsy. Grading by the House-Brackmann Scale showed that 6 patients were diagnosed as Grade II; 3 as Grade III; 14 as Grade IV; and 6 as Grade V.

\section{Differences in taste thresholds between Bell's palsy and control subjects}

EGM showed that the electrical taste thresholds at all five locations, four on the tongue and one on the central soft palate, were significantly higher in the Bell's palsy than in the control group ( $p<0.05$ each). Chemical tests for sweet, salty, bitter, sour, and savory tastes at the two locations on the tongue and central soft palate showed no significant differences in taste thresholds between the two groups $(p>0.05$ each) (Table 1).

\section{Taste thresholds as a function of severity} of facial paralysis

The Bell's palsy patients were divided into two subgroups based on the House-Brackmann Scale; a mild-moderate group (Grades II, III, and IV; $n=23$ ) and a severe group (Grades V and VI; n=6). No significant between-group differences in electrical taste thresholds or in chemical taste tests at any locations on the tongue and soft palate were observed ( $p>0.05$ each) (Table 2).

\section{Differences between Bell's palsy and control subjects aged $<40$ years}

Overall, the mean electrical taste thresholds in Bell's palsy patients aged $<40$ years were higher than in similarly aged control subjects, with the difference in the back-right area of the tongue differing significantly $(p<0.05)$. No significant differences in chemical taste thresholds were observed between these two groups ( $p>0.05$ each) (Table 3 ).

\section{Differences between Bell's palsy group and controls aged $>40$ years}

Overall, the mean electrical taste thresholds in Bell's palsy patients aged $>40$ years were significantly higher than in

Table 1. Taste threshold differences in the facial palsy and control groups

\begin{tabular}{|c|c|c|c|}
\hline & Control $(n=47)$ & Facial palsy $(n=29)$ & $p$-value \\
\hline \multicolumn{4}{|l|}{ Electrogustometry } \\
\hline Rt. Ant $(\mu \mathrm{A})$ & $22.48 \pm 59.72$ & $110.89 \pm 156.54$ & $0.002 *$ \\
\hline Rt. Post $(\mu \mathrm{A})$ & $48.57 \pm 114.40$ & $188.72 \pm 176.72$ & $0.002^{*}$ \\
\hline Lt. Ant $(\mu \mathrm{A})$ & $32.61 \pm 82.17$ & $148.2 \pm 186.31$ & $0.011 *$ \\
\hline Lt. Post $(\mu \mathrm{A})$ & $33.57 \pm 79.33$ & $165.82 \pm 179.62$ & $0.01^{*}$ \\
\hline$S P(\mu A)$ & $35.55 \pm 97.28$ & $147.62 \pm 177.94$ & $0.036^{*}$ \\
\hline \multicolumn{4}{|l|}{ Chemical taste tests } \\
\hline Sucrose-midline (M) & $0.33 \pm 0.48$ & $0.33 \pm 0.38$ & 0.435 \\
\hline NaCl-midline (M) & $0.37 \pm 0.31$ & $0.49 \pm 0.32$ & 0.113 \\
\hline Quinine-midline (M) & $0.01 \pm 0.01$ & $0.01 \pm 0.01$ & 0.969 \\
\hline Tartaric-midline $(M)$ & $0.14 \pm 0.15$ & $0.19 \pm 0.18$ & 0.138 \\
\hline MSG-midline (\%) & $0.33 \pm 0.26$ & $0.41 \pm 0.3$ & 0.282 \\
\hline Sucrose-SP (M) & $0.28 \pm 0.34$ & $0.2 \pm 0.15$ & 0.785 \\
\hline $\mathrm{NaCl}-\mathrm{SP}(M)$ & $0.36 \pm 0.3$ & $0.37 \pm 0.31$ & 0.815 \\
\hline Quinine-SP $(M)$ & $0.0096 \pm 0.016$ & $0.0051 \pm 0.009$ & 0.195 \\
\hline Tartaric-SP (M) & $0.1 \pm 0.1$ & $0.12 \pm 0.15$ & 0.724 \\
\hline MSG-SP (\%) & $0.28 \pm 0.22$ & $0.35 \pm 0.21$ & $0.042 *$ \\
\hline
\end{tabular}

* $p<0.05$. SP: soft palate, Rt.: right, Lt.: left, Ant: anterior, Post: posterior, MSG: monosodium glutamate 
Table 2. Taste thresholds in Bell's palsy patients according to the House-Brackmann Scale

\begin{tabular}{|c|c|c|c|}
\hline & Mild-moderate $(n=23)$ & Severe $(n=6)$ & $p$-value \\
\hline \multicolumn{4}{|l|}{ Electrogustometry } \\
\hline Rt. Ant $(\mu \mathrm{A})$ & $95.95 \pm 146.32$ & $168.16 \pm 195.11$ & 0.786 \\
\hline Rt. Post $(\mu \mathrm{A})$ & $180.82 \pm 173.88$ & $219 \pm 201.17$ & 0.46 \\
\hline Lt. Ant $(\mu \mathrm{A})$ & $115 \pm 173.5$ & $275.5 \pm 193.44$ & 0.091 \\
\hline Lt. Post $(\mu \mathrm{A})$ & $146.34 \pm 177.9$ & $240.5 \pm 181.64$ & 0.256 \\
\hline $\mathrm{SP}(\mu \mathrm{A})$ & $116.3 \pm 160.6$ & $267.66 \pm 205$ & 0.224 \\
\hline \multicolumn{4}{|l|}{ Chemical taste tests } \\
\hline Sucrose-midline (M) & $0.36 \pm 0.42$ & $0.22 \pm 0.14$ & 0.62 \\
\hline $\mathrm{NaCl}$-midline $(\mathrm{M})$ & $0.49 \pm 0.33$ & $0.46 \pm 0.34$ & 0.89 \\
\hline Quinine-midline (M) & $0.0082 \pm 0.01$ & $0.016 \pm 0.015$ & 0.23 \\
\hline Tartaric-midline $(M)$ & $0.16 \pm 0.13$ & $0.31 \pm 0.27$ & 0.319 \\
\hline MSG-midline (\%) & $0.42 \pm 0.28$ & $0.39 \pm 0.39$ & 0.681 \\
\hline Sucrose-SP (M) & $0.21 \pm 0.15$ & $0.16 \pm 0.13$ & 0.47 \\
\hline $\mathrm{NaCl}-\mathrm{SP}(M)$ & $0.38 \pm 0.29$ & $0.35 \pm 0.42$ & 0.564 \\
\hline Quinine-SP $(M)$ & $0.0049 \pm 0.008$ & $0.0059 \pm 0.011$ & 0.87 \\
\hline Tartaric-SP $(M)$ & $0.95 \pm 0.11$ & $0.22 \pm 0.22$ & 0.09 \\
\hline MSG-SP (\%) & $0.33 \pm 0.2$ & $0.43 \pm 0.23$ & 0.162 \\
\hline
\end{tabular}

Mild-moderate group, House Brackmann Grades II, III, and IV; severe group: House-Brackmann Grade V. Rt.: right, Lt.: left, Ant: anterior, Post: posterior, MSG: monosodium glutamate, SP: soft palate

Table 3. Differences in taste thresholds in Bell's palsy patients and control subjects aged $<40$ years

\begin{tabular}{|c|c|c|c|}
\hline & Control $(n=28)$ & Facial palsy $(n=11)$ & $p$-value \\
\hline \multicolumn{4}{|l|}{ Electrogustometry } \\
\hline Rt. Ant $(\mu \mathrm{A})$ & $12.42 \pm 20.22$ & $60.63 \pm 116.69$ & 0.094 \\
\hline Rt. Post $(\mu \mathrm{A})$ & $8.14 \pm 6.02$ & $110.18 \pm 146.03$ & $0.044^{*}$ \\
\hline Lt. Ant $(\mu \mathrm{A})$ & $14.57 \pm 24.49$ & $119.27 \pm 180.77$ & 0.099 \\
\hline Lt. Post $(\mu \mathrm{A})$ & $13.89 \pm 15.31$ & $118 \pm 155.82$ & 0.197 \\
\hline$S P(\mu A)$ & $10.07 \pm 15.18$ & $85.81 \pm 141.57$ & 0.289 \\
\hline \multicolumn{4}{|l|}{ Chemical taste tests } \\
\hline Sucrose-midline (M) & $0.2 \pm 0.16$ & $0.25 \pm 0.23$ & 0.551 \\
\hline NaCl-midline $(M)$ & $0.29 \pm 0.29$ & $0.3 \pm 0.3$ & 0.849 \\
\hline Quinine-midline (M) & $0.01 \pm 0.01$ & $0.01 \pm 0.01$ & 0.666 \\
\hline Tartaric-midline $(M)$ & $0.12 \pm 0.14$ & $0.11 \pm 0.09$ & 0.709 \\
\hline MSG-midline (\%) & $0.35 \pm 0.26$ & $0.37 \pm 0.3$ & 0.987 \\
\hline Sucrose-SP (M) & $0.18 \pm 0.18$ & $0.19 \pm 0.16$ & 0.664 \\
\hline $\mathrm{NaCl}-\mathrm{SP}(\mathrm{M})$ & $0.28 \pm 0.28$ & $0.21 \pm 0.2$ & 0.701 \\
\hline Quinine-SP $(M)$ & $0.0091 \pm 0.018$ & $0.0019 \pm 0.002$ & 0.252 \\
\hline Tartaric-SP $(M)$ & $0.08 \pm 0.11$ & $0.05 \pm 0.01$ & 0.249 \\
\hline MSG-SP (\%) & $0.3 \pm 0.23$ & $0.31 \pm 0.2$ & 0.404 \\
\hline
\end{tabular}

*p<0.05. Rt.: right, Lt.: left, Ant: anterior, Post: posterior, MSG: monosodium glutamate, SP: soft palate

similarly aged control subjects, but none of these differences was statistically significant ( $p>0.05$ ). No significant differences in chemical taste thresholds were observed between these two groups of older subjects $(p>0.05)$ (Table 4$)$.

\section{Discussion}

The most common cause of facial nerve paralysis is Bell's palsy, also known as idiopathic facial nerve palsy. Additional causes include trauma, herpes zoster oticus, tumors, infections, birth defects including congenital diseases, hemifacial spasm, lesions of the central nervous system, and atypical Bell's palsy. Less frequent causes include toxicities and metabolic and iatrogenic factors [8]. Bell's palsy can occur at any age, but its incidence is lowest in children under 10 years of age and older people [9] and highest in pregnant women 
Table 4. Differences in taste threshold in Bell's palsy patients and control subjects aged $\geq 40$ years

\begin{tabular}{|c|c|c|c|}
\hline & Control $(n=19)$ & Facial palsy $(n=18)$ & p-value \\
\hline \multicolumn{4}{|l|}{ Electrogustometry } \\
\hline Rt. Ant $(\mu \mathrm{A})$ & $37.31 \pm 90.06$ & $141.61 \pm 172.39$ & 0.053 \\
\hline Rt. Post $(\mu \mathrm{A})$ & $108.15 \pm 164.63$ & $236.72 \pm 180.18$ & 0.086 \\
\hline Lt. Ant $(\mu \mathrm{A})$ & $59.21 \pm 122.89$ & $165.88 \pm 192.56$ & 0.12 \\
\hline Lt. Post $(\mu \mathrm{A})$ & $62.57 \pm 119.34$ & $195.05 \pm 190.98$ & 0.053 \\
\hline$S P(\mu A)$ & $73.1 \pm 146.08$ & $185.38 \pm 190.74$ & 0.205 \\
\hline \multicolumn{4}{|l|}{ Chemical taste tests } \\
\hline Sucrose-midline $(M)$ & $0.54 \pm 0.69$ & $0.38 \pm 0.45$ & 0.95 \\
\hline NaCl-midline (M) & $0.5 \pm 0.32$ & $0.6 \pm 0.28$ & 0.31 \\
\hline Quinine-midline $(M)$ & $0.01 \pm 0.01$ & $0.01 \pm 0.01$ & 0.865 \\
\hline Tartaric-midline (M) & $0.16 \pm 0.16$ & $0.24 \pm 0.2$ & 0.223 \\
\hline MSG-midline (\%) & $0.3 \pm 0.26$ & $0.44 \pm 0.31$ & 0.147 \\
\hline Sucrose-SP (M) & $0.41 \pm 0.45$ & $0.21 \pm 0.14$ & 0.161 \\
\hline $\mathrm{NaCl}-\mathrm{SP}(\mathrm{M})$ & $0.48 \pm 0.31$ & $0.47 \pm 0.33$ & 0.963 \\
\hline Quinine-SP $(M)$ & $0.01 \pm 0.01$ & $0.007 \pm 0.01$ & 0.304 \\
\hline Tartaric-SP $(M)$ & $0.13 \pm 0.11$ & $0.16 \pm 0.17$ & 0.715 \\
\hline MSG-SP (\%) & $0.25 \pm 0.22$ & $0.37 \pm 0.22$ & 0.055 \\
\hline
\end{tabular}

Rt.: right, Lt.: left, Ant: anterior, Post: posterior, MSG: monosodium glutamate, SP: soft palate

[10], individuals with diabetes mellitus, and patients with a previous medical history of Bell's palsy [11]. Studies of symptoms accompanying Bell's palsy have found that, of patients with this condition, $67 \%$ have excessive tearing in the eyes, $52 \%$ have postauricular pain, 34\% have taste disorders, and $14 \%$ have phonophobia $[1,9]$.

Taste buds, which are distributed throughout the anteriortwo thirds of the tongue, are innervated by the chorda tympani and by special sensory nerve fibers of the facial nerve [12]. The posterior one-third of the tongue is innervated by the glossopharyngeal nerve, and taste buds of the soft palate are innervated by the greater petrosal nerve of the facial nerve. In this study, four locations on the tongue were tested, corresponding to the chorda tympani $(2 \mathrm{~cm}$ from the midline of the tongue apex laterally); and the glossopharyngeal nerves, including the circumvallate and foliate papillae. The central soft palate $1 \mathrm{~cm}$ from the palatine uvula was also tested. Since Bell's palsy patients develop unilateral facial paralysis, tongues were tested bilaterally [6].

Gustatory function tests can be classified as qualitative or quantitative based on their characteristics. EGM and chemical taste tests involve the administration of stimuli, whereas whole-mouth/local mouth tests are based on the locations of the tongue [5]. In this study, both EGM and chemical taste tests were performed throughout the entire mouth, thus better reflecting the taste sensations perceived during actual food ingestion. EGM showed that the electrical taste thresholds at five locations, four on the tongue and one on the central soft palate, were significantly higher in the Bell's palsy than in the control group. These results are consistent with previous reports showing that patients with Bell's palsy and herpes zoster oticus experience taste disorders, which are triggered by paralysis of the facial nerve [7,13]. EGM testing showed greater differences in taste thresholds between the Bell's palsy and control groups than did chemical taste tests. This finding suggests that EGM makes it easier to control stimulus strength and to objectively determine any mild taste disorder with few subjective symptoms [6,14].

The results of both EGM and chemical taste tests did not differ between patients with mild-moderate and severe Bell's palsy. These results suggest that normal or abnormal taste sensations at paralyzed sites are more important factors than the severity of taste disorders. Previous studies testing the effects of age on taste thresholds revealed that subjects aged $>40$ years had a higher threshold than those aged $<40$ years, although the magnitude of these differences between the two groups differed when assessing sour [15], sour/bitter [16], sweet [17], and sour/salty [18] tastes [19].

A study of Korean subjects showed that EGM thresholds increased significantly with age in both males and females [20]. Although a second study using chemical function tests found that all thresholds for sweet, salty, bitter and sour tastes increased, only the increase in salty taste threshold was statistically significant [21]. Changes in taste threshold with aging differed in men and women, with men showing gradual increases in taste thresholds from their $10 \mathrm{~s}$ to $60 \mathrm{~s}$, whereas women aged $<40$ years showed no differences and those aged $\geq 40$ tears experiencing radical changes [20]. Similarly, 
in this study, therefore, subjects aged $<40$ years with Bell's palsy showed significantly greater increases in EGM-determined taste thresholds than the control subjects. In patients aged $\geq 40$ years, the EGM-determined taste thresholds were higher in the Bell's palsy than in the control group, but the taste test-determined thresholds were not. This result suggested that taste sensitivity was similar in Bell's palsy patients and controls aged $>40$ years. Similar to the other senses (i.e., sight, hearing, smell and touch), taste loss may occur with age [19]. Many older patients take multiple medications because of chronic degenerative diseases [21], and taste function may be affected by factors such as gender [22], smoking [23], and medication [24]. At present, there are no widespread criteria for judging the presence of taste disorders at various ages [21].

The present study had several limitations. We could not compare changes in taste in Bell's palsy patients from before to after treatment or after stratifying patients by age or by intensity of Bell's palsy (House-Brackmann grade) due to the small sample size. In addition, we were unable to assess the relationship between EGM and chemical taste tests or the effects of previous medications, especially steroids, used to treat facial palsy.

In conclusion, the electrical taste thresholds were higher in patients with Bell's palsy than in healthy controls. In particular, the taste thresholds were higher among younger than older Bell's palsy patients.

\section{Acknowledgments}

This work was supported by the National Research Foundation of Korea (NRF) grant funded by the Korea government (MSIP) (No. 2011-0030072).

\section{Conflicts of interest}

The authors have no financial conflicts of interest.

\section{REFERENCES}

1) Lee HY, Ryu EW, Park SW, Kim SJ, Yeo SG, Park MS. Analysis of associated symptoms of Bell's palsy. Korean J OtorhinolaryngolHead Neck Surg 2011;54:683-7.

2) Birinyi F. Facial weakness and rash. Ramsay Hunt syndrome (herpes zoster cephalicus, herpes zoster oticus, herpes zoster auricularis). Acad Emerg Med 1996;3:1144-5, 1153-5.

3) Yamauchi Y, Endo S, Sakai F, Yoshimura I. A new whole-mouth gustatory test procedure. 1 . Thresholds and principal components analysis in healthy men and women. Acta Otolaryngol Suppl 2002;(546):
$39-48$.

4) Park SG, Kim SH, Kee WC, Choi JK. Changes in electrical taste threshold with advancing age in Korea. Korean J Oral Med 1998;23: 327-41.

5) Weiffenbach JM, Baum BJ, Burghauser R. Taste thresholds: quality specific variation with human aging. J Gerontol 1982;37:372-7.

6) Tomita H, Ikeda M. Clinical use of electrogustometry: strengths and limitations. Acta Otolaryngol Suppl 2002;(546):27-38.

7) Tomita H, Okuda Y, Tomiyama H, Kida A. Electrogustometry in facial palsy. Arch Otolaryngol 1972;95:383-90.

8) Terzis JK. Microreconstruction of nerve injuries. Part 5: combating facial paralysis. Philadelphia: WB Saunders; 1987.

9) Peitersen E. Bell's palsy: the spontaneous course of 2,500 peripheral facial nerve palsies of different etiologies. Acta Otolaryngol Suppl 2002;(549):4-30.

10) Holland NJ, Weiner GM. Recent developments in Bell's palsy. BMJ 2004;329:553-7.

11) Adour K, Wingerd J, Doty HE. Prevalence of concurrent diabetes mellitus and idiopathic facial paralysis (Bell's palsy). Diabetes 1975; 24:449-51.

12) Guyton AC, Hall JE. Textbook of medical physiology. 9th ed. Philadelphia: W.B. Sanuders. Co.;1996. p.676-7.

13) Krarup B. Taste reactions of patients with Bell's palsy. Acta Otolaryngol 1958;49:389-99.

14) Murphy C, Quiñonez C, Nordin S. Reliability and validity of electrogustometry and its application to young and elderly persons. Chem Senses 1995;20:499-503.

15) Yoshinaka M, Yoshinaka MF, Ikebe K, Shimanuki Y, Nokubi T. Factors associated with taste dissatisfaction in the elderly. J Oral Rehabil 2007;34:497-502.

16) Solemdal K, Sandvik L, Willumsen T, Mowe M. Taste ability in hospitalised older people compared with healthy, age-matched controls. Gerodontology 2014;31:42-8.

17) Kennedy O, Law C, Methven L, Mottram D, Gosney M. Investigating age-related changes in taste and affects on sensory perceptions of oral nutritional supplements. Age Ageing 2010;39:733-8.

18) Heft MW, Robinson ME. Age differences in orofacial sensory thresholds. J Dent Res 2010;89:1102-5.

19) Fukunaga A, Uematsu H, Sugimoto K. Influences of aging on taste perception and oral somatic sensation. J Gerontol A Biol Sci Med Sci 2005;60:109-13.

20) Park SG, Kim, SH. Kee WC, Choi JK. Changes in electrical taste threshold with advancing age in Korea. Korean J Oral Med 1998;23: 327-41.

21) Lee JW, Shin SH, Rhyu MR, Kim JY, Ye MK. The effect of aging on taste thresholds in Korean. Korean J Otorhinolaryngol-Head Neck Surg 2013;56:286-90.

22) Dangore-Khasbage SB, Degwekar SS, Bhowate RR, Motwani MB, Indurkar AD, Lohe VK, et al. Comparative evaluation of gustatory function between postmenopausal women and age-matched men. Oral Dis 2010;16:469-75.

23) Ye MK, Han BD, Lee JW, Rhyu MR, Hyun DS, Shin SH. Relationship between taste genotype and smoking and alcohol intake. Korean J Otorhinolaryngol-Head Neck Surg 2011;54:847-52.

24) Tomita H, Yoshikawa T. Drug-related taste disturbances. Acta Otolaryngol Suppl 2002;(546):116-21. 